\title{
A Systematic Study on Electronic and Thermodynamical Properties of Some LiM (M= Ag, $\mathrm{Hg}$ and $\mathrm{TI})$ Intermetallics
}

\author{
Neetu Paliwal ${ }^{1}$, Vipul Srivastava ${ }^{2, *}$ \\ ${ }^{1}$ Department of Physics, AISECT University, Bhopal, India \\ ${ }^{2}$ Department of Engineering Physics, NRI Institute of Research \& Technology, Bhopal, India
}

Email address:

vipsri27@gmail.com (V. Srivastava)

\section{To cite this article:}

Neetu Paliwal, Vipul Srivastava. A Systematic Study on Electronic and Thermodynamical Properties of Some LiM (M= Ag, Hg and Tl)

Intermetallics. International Journal of Materials Science and Applications. Vol. 4, No. 1, 2015, pp. 52-58. doi: 10.11648/j.ijmsa.20150401.20

\begin{abstract}
Binary intermetallic system, $\mathrm{Li}-\mathrm{M}(\mathrm{M}=\mathrm{Ag}, \mathrm{Hg}$ and $\mathrm{Tl})$ has been systematically evaluated and optimized using self consistent tight binding linear muffin tin orbital (TB-LMTO) method under ambient conditions. The lattice constant, bulk modulus and its pressure derivative were calculated. The lattice constants were calculated to be $3.05,3.20$ and $3.34 \AA$ and bulk modulii were predicted to be $61.8,50$ and $37.8 \mathrm{GPa}$, for LiAg, LiHg and LiTl, respectively. Electronic band structures, partial and total densities of states were derived in $\mathrm{B}_{2}(\mathrm{CsCl})$ phase for the first time. The band structures show metallic character and conductivity was mostly governed by Li-d and M-d states. Furthermore, Debye temperature, Grüneisen constant and molar heat capacity in terms of coefficients of the electronic and lattice heat capacities were estimated.
\end{abstract}

Keywords: TB-LMTO, Intermetallic Compounds, LiAg, LiHg, LiTl, Electronic Structure, Thermodynamical Properties

\section{Introduction}

Lithium belongs to the family of alkali metals and more closely resembles Group 2 (IIa) of the periodic table. The alkali metals have $n s^{1}$ valence electronic configuration. This single valence electron can be easily given to form a cation and therefore responsible for their attractive physical and chemical properties (high thermal and electrical conductivity) Lithium has the highest specific heat capacity of any solid element, and is therefore used in coolant for many heat transfer applications. Lithium compounds have a number of industrial applications, together with heat-resistant glass and ceramics, high strength-to-weight alloys used in aircraft, lithium batteries and lithium-ion batteries [1-3]. Nowadays, alkali-metal intermetallics have attracted more interest because of one of the unusual properties like deviation from Vegard's rule [4]. Considering the intermetallic system of $\mathrm{LiM}(\mathrm{M}=\mathrm{Ag}, \mathrm{Hg}$ and $\mathrm{Tl})$, to the best of our knowledge very little is known about the electronic and thermal properties. However, it is well known that LiM system adopts CsCl-type structure (B2-Phase; space group: 221). The crystal structure and phase diagram study of LiM is given elsewhere $[5,6]$. There are several binary intermmetallics fall in this category. Most of them fairly studied and understood with respect to their electronic band structure, density of states, specific heats and Debye temperature using ab initio theories [7-13]. The study of electronic specific heat coefficients of intermetallics has been interesting because of its direct relation with density of states at Fermi surface. Secondly, it has been observed in various cases [14-16] that electronic specific heat coefficient of individual metal is increased when alloyed.

The LiM intermetallics are motivated because (i) The LiM materials are new and electronic states are not well understood (ii) their possible thermal and electrical applications (iii) like other intermetallics probable increment in the electronic specific heat coefficient of constituent metal when alloying. Such lack of information has motivated us to explore the structural, electronic and thermodynamical properties of some LiM intermetallics by using combined the first principles tight binding linear muffin tin orbital (TB-LMTO) method and Debye-Grüneisen (DG) model.

The present paper is organized in such a way, methodology to calculate electronic states, Debye temperature and Grüneisen constant of all LiM intermetallics is briefly described in Section 2. Section 2.1 deals with the first principles computational details for describing electronic properties. The Debye temperature is evaluated incorporating first principles theory in DG model and briefly described in 
Section 2.2. The results with discussion on thermodynamical properties of all LiM intermetallics are presented in Section 3. Section 4 deals with the concluding remarks.

\section{Computation Methods}

\subsection{TB-LMTO Method}

The total energy, band structure and density of states for LiM intermetallics are calculated in nonmagnetic (NM) states, similar to our previous work [8,9] using TB-LMTO method with atomic sphere approximation (ASA) $[17,18]$ within the local-density approximation (LDA) [18]. von-Barth and Hedin [19] parameterization scheme had been used for exchange correlation potential. LiM intermetallics crystallize in the CsCl-type structure (space group, 221). The $\mathrm{CsCl}$ structure has a body centre cubic structure with one formula unit per unit cell ${ }^{3}$.

Lattice: Body centered cubic (BCC) and

Basis: $\mathrm{Li}$ at $(0,0,0)$ and $\mathrm{M}$ at $(1 / 2,1 / 2,1 / 2)$.

The Wigner-Seitz sphere was chosen in such a way that the boundary potential was minimum and charge flow between the atoms was in accordance with the electro-negativity criteria. The $\mathrm{E}$ and $\mathrm{K}$ convergence were also checked. The tetrahedron method [20] of Brillouin zone integration had been used to calculate the total density of states. We have used $8 \mathrm{x} 8 \mathrm{x} 8 \mathrm{~K}$ grid $(512 \mathrm{~K}$ points) in the Brilliuon zone. To find the equilibrium lattice constant, the total energies are computed by changing the volume from $1.05 V_{0}$ to $0.65 V_{0}$ using Birch of state [21].

$$
P=\frac{3}{2} B_{0}\left[\left(V / V_{0}\right)^{-7 / 3}-\left(V / V_{0}\right)^{-5 / 3}\right]\left[1+\frac{3}{4}\left(B_{0}^{\prime}-4\right)\left(\left(V / V_{0}\right)^{-2 / 3}-1\right)\right]
$$

where $P$ is the pressure, $V$ is the volume at $P, V_{0}$ is the equilibrium cell volume, $B_{0}$ is the bulk modulus, and $B_{0}^{\prime}$ is the first order pressure derivative at equilibrium. Furthermore, the bulk modulus is calculated using the relation.

$$
B=-V_{0}\left(\frac{d P}{d V}\right)
$$

\subsection{Debye Model and Molar Heat Capacity}

The thermodynamical properties like Debye temperature and Grüneisen constant of a vibrating Debye lattice were calculated using Debye-Grüneisen (DG) model [22]. Debye temperature $\Theta_{D}$ was calculated using the following expression

$$
\Theta_{D}=41.63 \sqrt{\frac{r_{0} B}{M}}
$$

where $\mathrm{r}$ is Wigner radius in a.u., B is bulk modulus in kbar and $\mathrm{M}$ is atomic weight. The calculated value of $\left(\Theta_{D}\right)_{0}$ using above expression, at $r=r_{0}$ (at equilibrium) and substituting the calculated (or experimental) value of bulk modulus deviates from the experimental value to a larger from the experimental value. To overcome from this situation a scaling factor had been introduced. In this modified expression, the theoretical value of $\mathrm{r}$ at $\mathrm{r}=\mathrm{r}_{0}$ and bulk modulus derived from the present first principles calculations at $r_{0}$ were substituted and the Debye temperature $\left(\Theta_{D}\right)_{0}$ was calculated using the expression

$$
\Theta_{D}=41.63 \sqrt{\frac{r_{0} B}{M}}
$$

and Grüneisen constant $\alpha$ was calculated by

$$
\alpha=\frac{\partial \ln \Theta_{D}}{\partial \ln V}
$$

where $\mathrm{V}$ is the volume of the solid.

The calculations on electronic structure and density of states enable us to estimate the molar heat capacity, $C_{p}$ at the low-temperatures. Therefore, $C_{p}$ was calculated in terms of electronic and lattice heat capacities as:

$$
C p(T)=\gamma T+\beta T^{3}
$$

where $\gamma=\frac{\pi^{2} K_{B}^{2} N\left(E_{f}\right)}{3} ; \beta=\frac{12 \pi^{4} R n}{5 \Theta_{D}^{3}}$

In (6), $\gamma$ and $\beta$ are the coefficients of electronic and lattice heat capacities, respectively; $K_{B}$ is Boltzman constant, $R$ is the molar gas constant, $n$ is the total number of atoms per formula unit. In condensed matter physics, the calculation of coefficient of electronic specific heat provides information about DOS at Fermi surface. Again the information on Fermi surface of the solids has been useful tool to predict various properties of solids like thermodynamical, electrical, magnetic, and optical properties. There have been extensive studies reported on specific heat calculations of some intermetallics in the literature [14-16].

\section{Results and Discussion}

In order to draw attention on the electronic and thermodynamical properties like band structure, density of states, bulk modulus, Debye temperature and heat coefficients of some Li based intermetallics, we have computed total energy using the approach described in above section. The total energy is plotted with different compressions for $\mathrm{LiAg}$, $\mathrm{LiHg}$ and LiTl and depicted in Figure 1.

We have found equilibrium volume $V_{0}$ (or equilibrium separation, $r_{0}$ ), at $28.37 \AA^{3}$ for $\mathrm{LiAg}$ and the corresponding lattice parameter $a, 3.05 \AA$ (5.763 a.u.). Similarly, other LiM intermetallics estimate equilibrium volume at, 32.82 and $37.42 \AA^{3}$ and the corresponding lattice parameter values 3.20 $\AA$ (6.050 a.u.) and $3.34 \AA$ (6.320 a.u.) for $\mathrm{LiHg}$ and $\mathrm{LiTl}$, respectively, which are tabulated in Table 1 . We have compared our results with the experimental results [6]. A good agreement is established. We have also calculated bulk modulii as $61.8,50$, and $37.5 \mathrm{GPa}$ and pressure derivatives as 3.6, 3.7 and 3.9 for $\mathrm{LiAg}, \mathrm{LiHg}$ and $\mathrm{LiTl}$ intermetallics under ambient conditions, respectively. LiAg found to be stiffer than LiTl. 

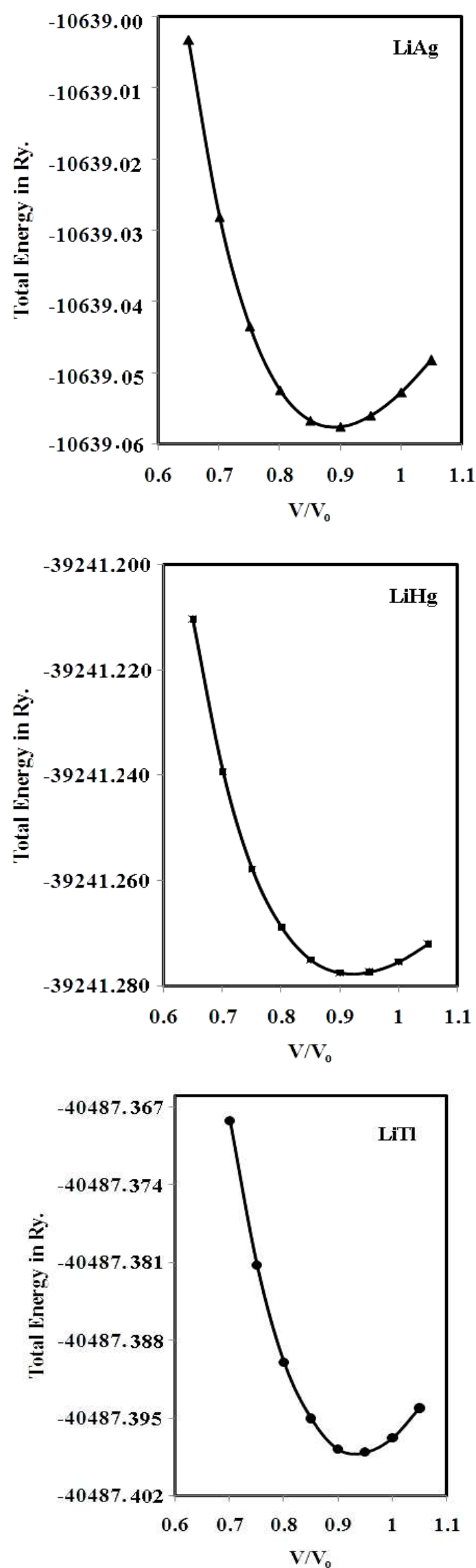

Figure 1. Variation of total energy as a function of relative volume for LiM $(M=A g, H g$ and $T l)$ intermetallics
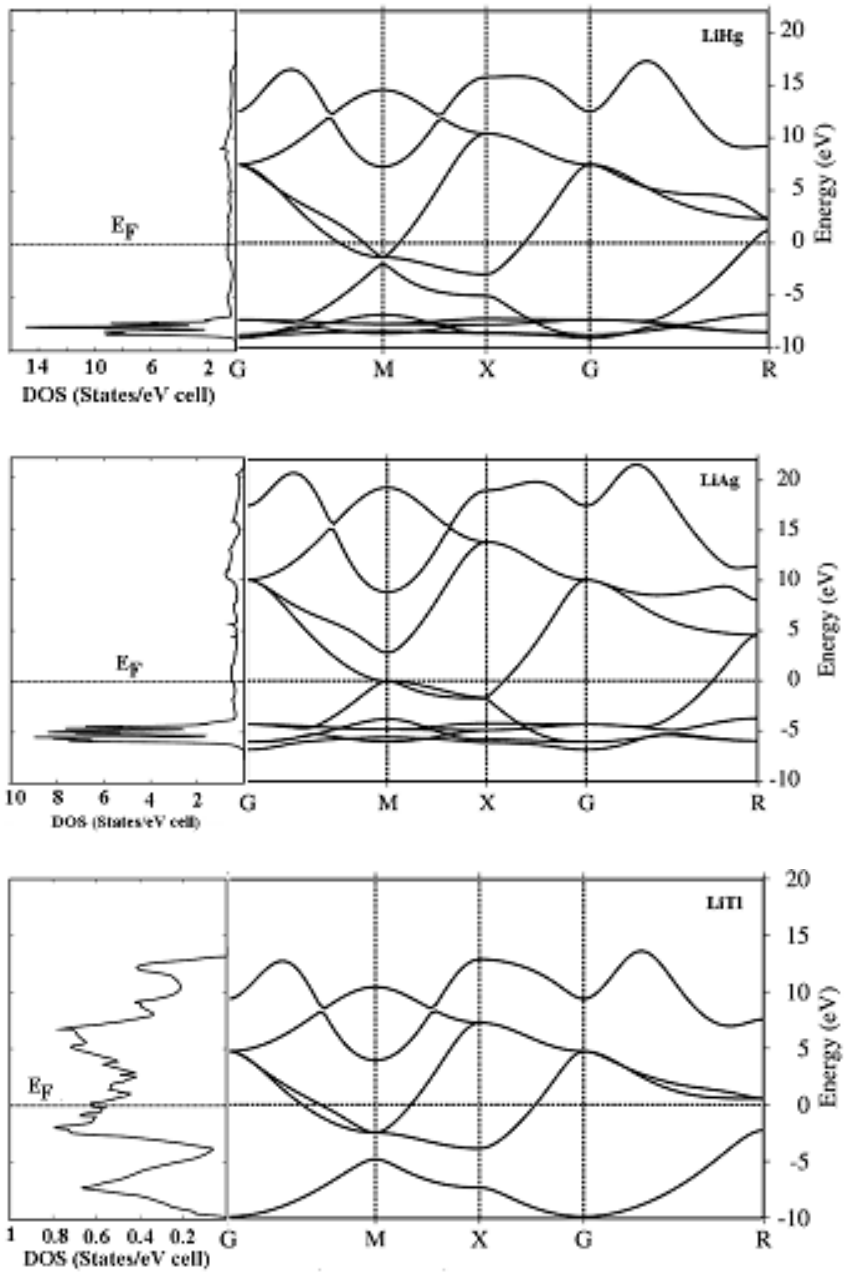

Figure 2. Band structure along the high symmetry directions with total density of states at $E_{f}$ under ambient conditions for LiM intermetallics .

We have plotted a combined picture of self consistent band structures along the high symmetry directions and total density of states at Fermi level for all the LiM intermetallics in Figure 2. It is seen from the Figure 2 that lowest lying bands in these compounds are mainly due to the $\mathrm{M}-$ ' $\mathrm{s}$ ' $(\mathrm{M}=\mathrm{Ag}, \mathrm{Hg}$ and $\mathrm{Tl}$ ) like states. The top most band is basically the Li- 's' like states. However, M-'d' and M-'p' like states can be seen crossing over Fermi level, $\mathrm{E}_{\mathrm{f}}$ and hybridizing with the Li- ' $\mathrm{p}$ ' like states and making them intermetallic. M-'d' like states can be seen between energy range of $-5 \mathrm{eV}$ to $-10 \mathrm{eV}$. The same can be understood by considering the total density of states at Fermi level, plotted with band structures.

The valence electrons of each element of LiM intermetallics are considered as:

Li: $1 s^{2} 2 s^{1}$

Ag: $[K r] 4 d^{10} 5 s^{1}$

Hg: [Xe] $4 \mathrm{f}^{14} 5 \mathrm{~d}^{10} 6 \mathrm{~s}^{2}$

Tl: $[\mathrm{Xe}] 4 \mathrm{f}^{14} 5 \mathrm{~d}^{10} 6 \mathrm{~s}^{2} 6 \mathrm{p}^{1}$

In order to understand the clear depiction of the elemental contributions to the electronic structure we have calculated 
partial density of states (PDOS) of LiM intermetallics and depicted in Figure 3. In $\mathrm{LiAg}$, the peak corresponds to the DOS value of 0.4 states/eV cell predominantly due to Li-' $\mathrm{p}$ ' like states, which falls at the same energy scale (hybridization) of Ag-'p' and Ag-'d' likes states.

Table 1. The calculated lattice parameter $a_{0}\left(\right.$ a.u.), bulk modulus $B_{0}(G P a)$, density of states at Fermi level N(E $\left.E_{f}\right)$ (states/Ry./f.u.), electronic heat co-efficient $\gamma$ $\left(\mathrm{J} / \mathrm{mol} \mathrm{K}^{2}\right)$, lattice heat co-efficient $\beta\left(\mathrm{J} / \mathrm{K}^{4} \mathrm{~mol}\right)$ Wigner-seitz radius $r_{0}($ a.u. $)$, Debye temperature $\left(\Theta_{D}\right)_{0}(\mathrm{~K})$ and $\mathrm{Grüneisen} \mathrm{constants} \alpha_{0}$ for LiM $(M=A g, H g$ and $T l)$ under ambient conditions.

\begin{tabular}{|c|c|c|c|c|c|c|c|c|}
\hline Solid & $a_{0}$ & $\boldsymbol{B}_{0}$ & $N\left(E_{f}\right)$ & $\gamma\left(10^{-3}\right)$ & $\beta\left(10^{-5}\right)$ & $\mathbf{r}_{0}$ & $\Theta_{\mathrm{D}}$ & $\alpha$ \\
\hline $\mathrm{LiAg}$ & $\begin{array}{l}5.763 \\
5.990^{\mathrm{a}}\end{array}$ & 61.8 & 6.85 & 1.04 & 31.9 & 2.837 & 229.8 & 1.19 \\
\hline $\mathrm{LiHg}$ & $\begin{array}{l}6.050 \\
6.217^{\mathrm{a}}\end{array}$ & 50.0 & 6.72 & 1.16 & 98.3 & 2.979 & 157.9 & 1.07 \\
\hline LiTl & $\begin{array}{l}6.320 \\
6.463^{\mathrm{a}}\end{array}$ & 37.5 & 8.42 & 1.45 & 146.3 & 3.112 & 138.4 & 1.02 \\
\hline
\end{tabular}

${ }^{\text {a }}$ Ref.[6].
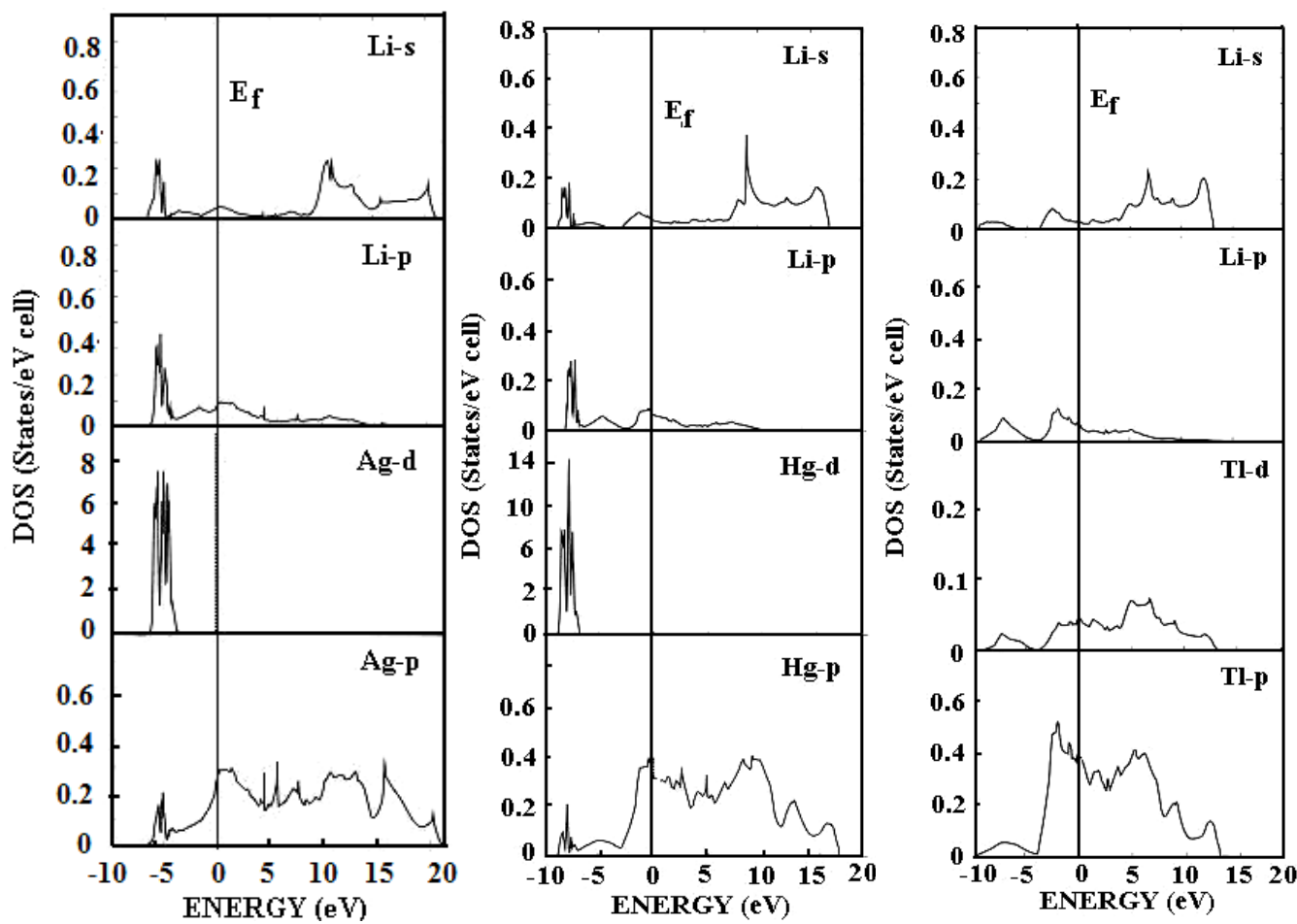

Figure 3. Partial density of states at Fermi level for LiM intermetallics.

Ag-'p' and Ag-'d' like states correspond to DOS peak value of 0.3 and 7.8 states/eV cell, respectively. In $\mathrm{LiHg}$, the peak corresponds to the DOS value of 0.3 states/eV cell is mainly due to Li-'p' like states, which falls similarly, at the same energy scale of Hg-'p' and Hg-'d' likes states with DOS peak value of 0.38 and 14 states/eV cell. Similarly, in LiTl, the DOS peak corresponds to the value of 0.17 states/eV cell due to Li- 'p' like states, which hybridize with Tl-'p' and Tl-'d' likes states with DOS peak corresponds to the value of 0.58 and 0.08 states/eV cell, respectively. The DOS value of 0.08 states/eV cell is very small as compare to other members of the this family. One can understand the reason of increase and then decrease of DOS value (d-states) in the present three intermetallics as: in the periodic table atomic number increases from $\mathrm{Ag}$ to $\mathrm{Tl}$ or on the otherhand, the number of d-electrons increases. The DOS value of 7.8 states/eV calculated for $\mathrm{LiAg}$, increases to $\sim 14$ states/eV for $\mathrm{LiHg}$, which in case of LiTl starts decreasing to a value of $\sim 0.08$
states/eV. The decrease in DOS could be the reason that d-electrons in $\mathrm{Tl}$ get delocalized even at ambient pressure. Such delocalization one can understand from PDOS diagram (Figure 3) of LiTl.

The partial number of electrons and total density of states at $\mathrm{E}_{\mathrm{f}}$ are also calculated under compression and presented in Table 2-4. One can notice the general valence transfer into the various states under compression. Also, DOS at $\mathrm{E}_{\mathrm{f}}$ decreases under compression, which is a quit general trend. In fact, under compression valence electrons are displaced, consequently DOS changes with respect to energy at the Fermi surface, which in turn directly related to the electronic specific heat. The value of DOS at $\mathrm{E}_{\mathrm{f}}$ for $\mathrm{LiAg}$ decreases from 6.90 to 5.93 states/Ry. cell at compression value of $\mathrm{V} / \mathrm{V}_{0}=1.05-0.65$ (Table 2). In case of $\mathrm{LiHg}$ such decrease in DOS at $\mathrm{E}_{\mathrm{f}}$ is noticed from 7.57 to 4.95 states/Ry. cell between compression value of $\mathrm{V} / \mathrm{V}_{0}=1.05-0.65$ (Table 3 ). As far as, such variation for LiTl is concerned, the decrease in DOS is noticed from 
9.91 to 6.28 states/Ry. cell at $\mathrm{E}_{\mathrm{f}}$ between compression value of $\mathrm{V} / \mathrm{V}_{0}=1.05-0.70$, while small increase in DOS is noticed to 8.12 states/Ry. cell at $\mathrm{V} / \mathrm{V}_{0}=0.65$ (Table 4). Such increase in DOS is noticed only in LiTl could be the indication of structural instability under compression.

In order to get the information regarding thermodynamical properties of such intermetallic system, we have calculated the Debye temperature $\left(\Theta_{\mathrm{D}}\right)_{0}$, Grüneisen constants $\alpha_{0}$ and molar heat capacity $C_{p}$ in terms of co-efficients of electronic and lattice heat capacities. The Debye temperature and Grüneisen constants are calculated using (4) and (5) taken from DG model [23]. The model describes $\Theta_{\mathrm{D}}$, can be obtained from the calculated values of bulk modulus, whereas $\alpha$ can be obtained from $\Theta_{D}$ over a volume, as expressed in previous section. The bulk modulus $\mathrm{B}_{0}$, Wigner-Seitz radii $\mathrm{r}_{0}$, Debye temperature $\left(\Theta_{\mathrm{D}}\right)_{0}$ and Grüneisen constants $\alpha_{0}$ at absolute temperature are calculated and presented in Table 1 .

Table 2. Calculated partial number of electrons for $\mathrm{LiAg}$

\begin{tabular}{|c|c|c|c|c|c|c|c|c|}
\hline \multirow{2}{*}{$\mathbf{V} / \mathbf{V}_{\mathrm{o}}$} & \multicolumn{3}{|c|}{ Partial number of electrons $\mathrm{Li}$} & \multicolumn{4}{|c|}{ Partial number of electrons Ag } & \multirow{2}{*}{ DOS (States/ Ry cell) } \\
\hline & $\mathbf{s}$ & $\mathbf{p}$ & d & $\mathbf{s}$ & $\mathbf{p}$ & d & f & \\
\hline 1.05 & 0.336 & 0.500 & 0.115 & 0.913 & 0.500 & 9.607 & 0.025 & 6.90 \\
\hline 0.95 & 0.329 & 0.513 & 0.116 & 0.900 & 0.544 & 9.565 & 0.031 & 6.96 \\
\hline 0.90 & 0.323 & 0.519 & 0.115 & 0.895 & 0.569 & 9.542 & 0.034 & 6.87 \\
\hline 0.85 & 0.317 & 0.525 & 0.115 & 0.890 & 0.595 & 9.516 & 0.038 & 6.73 \\
\hline 0.80 & 0.312 & 0.532 & 0.114 & 0.884 & 0.624 & 9.488 & 0.043 & 6.56 \\
\hline 0.75 & 0.304 & 0.537 & 0.114 & 0.879 & 0.657 & 9.457 & 0.049 & 6.37 \\
\hline 0.70 & 0.297 & 0.543 & 0.113 & 0.873 & 0.692 & 9.423 & 0.056 & 6.16 \\
\hline 0.65 & 0.292 & 0.549 & 0.113 & 0.865 & 0.734 & 9.382 & 0.064 & 5.93 \\
\hline
\end{tabular}

Table 3. Calculated partial number of electrons for $\mathrm{LiHg}$

\begin{tabular}{|c|c|c|c|c|c|c|c|c|}
\hline \multirow{2}{*}{$\mathbf{V} / \mathbf{V}_{\mathbf{o}}$} & \multicolumn{3}{|c|}{ Partial number of electrons $\mathrm{Li}$} & \multicolumn{4}{|c|}{ Partial number of electrons $\mathrm{Hg}$} & \multirow{2}{*}{ DOS (States/ Ry cell) } \\
\hline & $\mathbf{s}$ & $\mathbf{p}$ & d & $\mathbf{s}$ & $\mathbf{p}$ & d & f & \\
\hline 1.05 & 0.333 & 0.487 & 0.115 & 1.375 & 0.868 & 9.774 & 0.032 & 7.57 \\
\hline 1.00 & 0.325 & 0.493 & 0.115 & 1.358 & 0.897 & 9.760 & 0.035 & 7.24 \\
\hline 0.95 & 0.318 & 0.500 & 0.116 & 1.340 & 0.927 & 9.744 & 0.039 & 6.91 \\
\hline 0.90 & 0.311 & 0.508 & 0.115 & 1.320 & 0.959 & 9.726 & 0.043 & 6.57 \\
\hline 0.85 & 0.303 & 0.514 & 0.115 & 1.301 & 0.996 & 9.707 & 0.049 & 6.24 \\
\hline 0.80 & 0.296 & 0.523 & 0.114 & 1.277 & 1.035 & 9.684 & 0.055 & 5.94 \\
\hline 0.75 & 0.286 & 0.531 & 0.114 & 1.246 & 1.087 & 9.660 & 0.063 & 5.62 \\
\hline 0.70 & 0.276 & 0.538 & 0.113 & 1.222 & 1.132 & 9.632 & 0.073 & 5.29 \\
\hline 0.65 & 0.267 & 0.540 & 0.113 & 1.199 & 1.184 & 9.602 & 0.084 & 4.95 \\
\hline
\end{tabular}

Table 4. Calculated partial number of electrons for LiTl

\begin{tabular}{|c|c|c|c|c|c|c|c|c|}
\hline \multirow{2}{*}{$V / V_{o}$} & \multicolumn{3}{|c|}{ Partial number of electrons $\mathbf{L i}$} & \multicolumn{4}{|c|}{ Partial number of electrons TI } & \multirow{2}{*}{ DOS (States/ Ry cell) } \\
\hline & $\mathbf{s}$ & $\mathbf{p}$ & d & $\mathbf{s}$ & $\mathbf{p}$ & d & f & \\
\hline 1.05 & 0.298 & 0.448 & 0.093 & 1.544 & 1.455 & 0.131 & 0.027 & 9.91 \\
\hline 0.95 & 0.283 & 0.456 & 0.090 & 1.501 & 1.484 & 0.152 & 0.032 & 8.85 \\
\hline 0.90 & 0.274 & 0.461 & 0.089 & 1.474 & 1.500 & 0.165 & 0.034 & 8.01 \\
\hline 0.85 & 0.264 & 0.463 & 0.087 & 1.446 & 1.518 & 0.181 & 0.037 & 7.46 \\
\hline 0.80 & 0.254 & 0.467 & 0.085 & 1.415 & 1.536 & 0.200 & 0.040 & 6.95 \\
\hline 0.75 & 0.242 & 0.468 & 0.082 & 1.381 & 1.556 & 0.223 & 0.044 & 6.38 \\
\hline 0.70 & 0.231 & 0.470 & 0.079 & 1.344 & 1.574 & 0.251 & 0.058 & 6.28 \\
\hline 0.65 & 0.220 & 0.470 & 0.076 & 1.304 & 1.592 & 0.283 & 0.053 & 8.12 \\
\hline
\end{tabular}

We couldn't compare our results for the want of the experimental and theoretical data. However calculated value of Debye temperature for $\mathrm{LiAg}$ can be compared with the other silver-zinc alloys [15] and found to be in accordance. The thermodynamical properties of several other $B_{2}$ phase binary intermetallics have been calculated and available in literature [23-28].
We have performed variation of $\theta_{D}$ with compression and depicted in Figure 4. It shows $\theta_{D}$ increases under compression for all the cases, which is quite natural. No decrease in $\theta_{D}$ is noticed under compression. It can be noted that $\theta_{D}$ only explains the behavior of $C_{p}$ under temperature, therefore in Figure 5, we plot molar heat capacity, $C_{p}$ as a function of temperature (range of 0-15 K). 


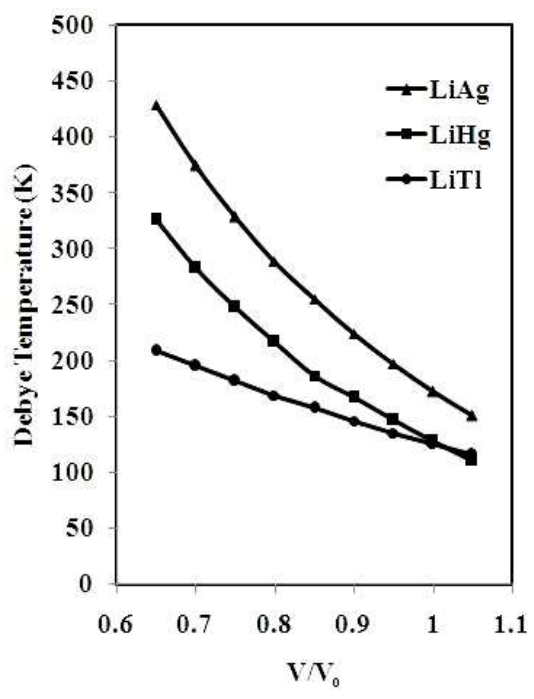

Figure 4. Variation of Debye temperature under compression for LiM intermetallics.

Figure 5 reveals that in the temperature range of $0-3 \mathrm{~K}$, the main contribution to $C_{p}$, comes entirely from the excitation of electrons. LiAg has the lowest value of $C_{p}$ which is in accordance with the calculated value of electronic heat capacity and resembles that $C_{p}$ determined by the electrons. As can be seen from the (6) that electronic heat capacity depends on DOS and maximum the value of DOS, maximum the value of electronic heat capacity. Tables 2-4 show DOS values at $E_{f}$ and one can notice the sequence of $\gamma$ in LiM as $\mathrm{LiAg}<\mathrm{LiHg}<\mathrm{LiTl}$.

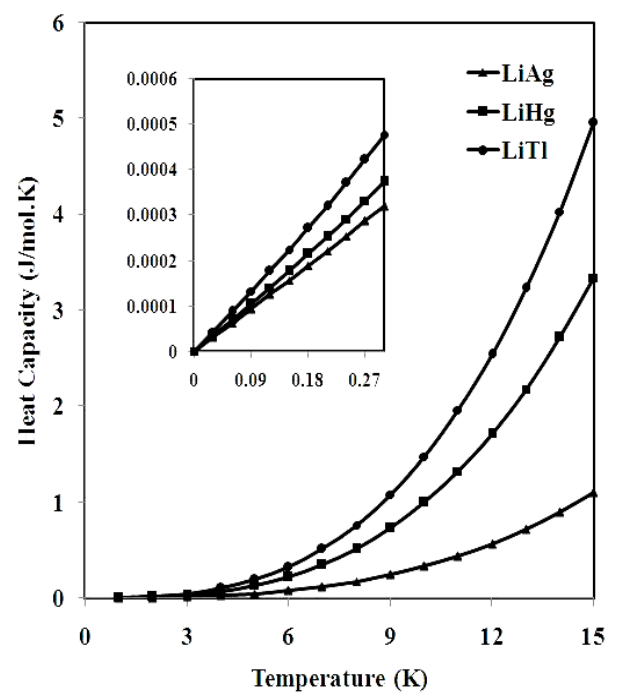

Figure 5. Heat capacity of LiM intermetallics as a function of temperature. Inset shows variation in Molar heat capacity under low temperature.

Further, Figure 5 depicts that in the temperature range of $3-15 \mathrm{~K}$, phonon excitations take place, which can be pointed out from the variation of $C_{p}$ (same as $\beta$ ). As a result, heat capacity is determined by the electron excitations at low temperature $(0-3 \mathrm{~K})$, shown in figure as an inset and phonon excitations are noteworthy at high temperatures $(3-15 \mathrm{~K})$. Since there are no experimental observations available to the best of our knowledge, hence a comparison is not possible.

\section{Conclusions}

A systematic theoretical study on calculations on electronic and thermodynamical properties of some $\mathrm{LiM}(\mathrm{M}=\mathrm{Ag}, \mathrm{Hg}$ and $\mathrm{Tl}$ ) intermetallics is presented using TB-LMTO and DG model, respectively. The main motive of this study was to get the technologically important information of LiM intermetallics in terms of various physical properties such as DOS, bulk modulli, pressure derivatives, Debye temperature, Grüneisen constant and molar heat capacities. All the LiM intermetallics stable in CsCl-type structure, which is in agreement with experimental work. It is found from the present study that LiM intermetallics are metallic in nature. We found metallic bonding between Li- and M- atoms under ambient conditions. The electronic band structures and DOS diagrams confirm their metallic nature. DOS under compression also obtained and found normal deceasing trend, except in LiTl at compression value of $65 \%$ (high pressure). The increase in DOS at $\mathrm{V} / \mathrm{V}_{0}=0.65$ is noticed only in LiTl, could be the indication of structural instability under compression. We have used Debye-Grüneisen model to calculate Debye temperature and Grüneisen constant. So far, no experimental and theoretical studies have been reported in the literature on electronic and thermodynamical properties of present LiM intermetallics, we therefore, present our results and open a scope for the physicists.

\section{Acknowledgements}

NP is thankful to Prof. V. K. Verma, Vice Chancellor, AISECT University, Bhopal, India for his constant support and encouragement. VS is thankful to Shri D. Subodh Singh, Chairman, NRI Group of Institutions (NGI) and Prof. G. Kumar, Director, NRI Institute of Research \& Technology (NIRT), Bhopal, India. VS acknowledges Madhya Pradesh Council of Scientific \& Technology (MPCST), Bhopal, India for the award of Research Project. Authors like to give especial thank to Prof. S. P. Sanyal, Department of Physics, Barkatullah University, Bhopal, India and Dr. A. K. Bandyopadhyay, Pressure Standards Laboratory, National Physical Laboratory (NPL), New Delhi, India for valuable discussion.

\section{References}

[1] H. Abe, T. Murai,; K. Zaghib, Journal of Power Sources 77 (2) (1999) 110.

[2] X. Zhao, C. M. Hayner, M. C. Kung, H. H. Kung, Advanced Energy Materials 1 (6) (2011) 1079.

[3] Y. Tang, Y. Zhang, J. Deng, J. Wei, H L Tam, B. K. Chandran, Z. Dong, Z. Chen and X. Chen, Advanced Materials, 26 (2014) 6046 .

[4] Z. H. Yu, C. Y. Li and H. Z. Liu, Physica B Condensed Matter, 407 (2012) 805.

[5] Desk Handbook: Phase Diagrams for Binary Alloys edited by Hiroaki Okamoto and A D Pelton, Bull. Alloy Phase Diagrams, 7 (1986) 223. 
[6] P. Villars and L. D. Calvert, Pearson's Handbook of Crystallographic Data for Intermetallic Phases, Vols. 1-4, ASM International, Materials Park, OH. (1991).

[7] H. Devi, G. Pagare, S. S. Chouhan, S. P. Sanyal Journal of Physics and Chemistry of Solids 76 (2015) 70.

[8] A. A. Khan, V. Srivastava, M. Rajagopalan and S. P. Sanyal, American Journal of Physics and Application, Vol. 2, (2014) 156.

[9] V. Srivastava, A. A.. Khan and S. P. Sanyal Physica B Condensed Matter, 407 (2012) 1873.

[10] J. Teeriniemi, P. Taskinen, K. Laasonen Intermetallics, 57 (2015) 41.

[11] Ş. Uğur, G. Uğur, F. Soyalp, M.R. Ellialtıŏlu, Intermetallics, $22(2012) 218$.

[12] SHI Yao-jun1, DU Yu-lei, CHEN Guang, Trans. Nonferrous Met. Soc. China 22 (2012) 654.

[13] G.. Pagare, V. Srivastava, S. P. Sanyal, and M. Rajagopalan, Physica. B Condensed Matter, 406 (2011) 449.

[14] Ben A. Green, Jr and Harvey V. Culbert, Physical Review A, 137 (1965) 1168.

[15] Ben A. Green, Jr, Specific Heats of AgZn Alloys, 144 (1965) 528.

[16] Lavern C. Clune and Ben A. Green Jr, Physical Review, 144 (1966) 525.
[17] O K Andersen, Phys. Rev. B12 (1975) 3060.

[18] O. K. Andersen and O. Jepsen, Phys. Rev. Lett. 53 (1984) 2571.

[19] U. van Barth and L. Hedin, J. Phys. C5, 1629 (1972).

[20] O. Jepsen and O. K. Andersen, Solid State Commun. 9, 1763 (1971).

[21] F. Birch, J. Geophys. Rev. 83 (1978) 1257.

[22] M. A. Blano, E. Francisco and V. Luana, Computer Physics Communication 158 (2004) 57.

[23] X. Tao, Y. Ouyang, H. Liu, F. Zeng, Y. Feng and Z. Jin, Computational Materials Science 40 (2007) 226;

[24] Q. Chen, Z. Huang, Z. Zhao and C. Hu, Computational Materials Science, 67 (2013) 196.

[25] Y-B. Kang, L. Jin, P. Chartrand, A. E. Gheribi, K. Bai and P. Wu, CALPHAD: Computer Coupling of Phase Diagrams and Thermochemistry, 38 (2012) 100.

[26] C. Yan, L. Lin, W. Yue-hui, L. Jian-hua and Z. Rui-jun Trans. Nonferrous Met. Soc. China 21 (2011) 2205.

[27] H. Jones, Proc. Roy. Soc. (London) A 240 (1957) 321.

[28] J. A. Rayne and W. R. G. Kemp, Australian J. Phys. 9 (1956) 569. 